\title{
Clinical Trial Roles and Responsibilities Matrix
}

National Cancer Institute

\section{Source}

National Cancer Institute. Clinical Trial Roles and Responsibilities Matrix. NCI Thesaurus. Code C125429.

A document describing the overall management strategy for vendors used to conduct trial-related activities. 